\title{
Novel molecular evidence of population structure in Anopheles (Kerteszia) bellator from Brazilian Atlantic Forest
}

\author{
Kamila Voges ${ }^{1}$, Marcela Possato Correa da Rosa', Betina Westphal-Ferreira ${ }^{2}$, \\ Mario Antonio Navarro-Silva², Carime Lessa Mansur Pontes ${ }^{3}$, André Nóbrega Pitaluga ${ }^{4,5,6}$, \\ Carlos José de Carvalho-Pinto ${ }^{3,6}$, Luísa DP Rona ${ }^{1,5,6 /+}$
}

${ }^{1}$ Universidade Federal de Santa Catarina, Centro de Ciências Biológicas, Departamento de Biologia Celular, Embriologia e Genética, Florianópolis, SC, Brasil

${ }^{2}$ Universidade Federal do Paraná, Setor de Ciências Biológicas, Departamento de Zoologia, Curitiba, PR, Brasil

${ }^{3}$ Universidade Federal de Santa Catarina, Centro de Ciências Biológicas, Departamento de Microbiologia, Imunologia e Parasitologia,

Florianópolis, SC, Brasil

${ }^{4}$ Fundação Oswaldo Cruz-Fiocruz, Instituto Oswaldo Cruz, Laboratório de Biologia Molecular de Parasitas e Vetores,

Rio de Janeiro, RJ, Brasil

${ }^{5}$ Imperial College London, Department of Life Sciences, London, United Kingdom

${ }^{6}$ Instituto Nacional de Ciência e Tecnologia em Entomologia Molecular, Rio de Janeiro, RJ, Brasil

Anopheles bellator is a primary malaria vector in the Atlantic Forest. Partial sequences of timeless and Clock genes were used to assess the genetic differentiation of five Brazilian populations, which showed strong population structure (e.g. high $F_{S T}$ values and fixed differences) in all pairwise comparisons between Bahia sample and the others from Paraná, São Paulo and Rio de Janeiro states. Also, the resulting phylogenetic trees clearly grouped the sequences from Bahia in a different cluster with high bootstrap values. Among southern and southeastern populations low levels of genetic differentiation were found suggesting a general stability of the genetic structure.

Key words: Anopheles (Kerteszia) bellator - bromeliad-malaria - Brazilian Atlantic Forest

Anopheles (Kerteszia) bellator, a mosquito species whose immature stages develop in water accumulated in bromeliads, occurs in Trinidad and Tobago and coastal areas spanning eastern Venezuela to southern Brazil. ${ }^{(1,2)}$ Interestingly, it is only considered a main human malaria vector in the two extremes of its distribution area ${ }^{(3,4)}$ and despite its epidemiological importance, there are few molecular studies concerning this species. Carvalho-Pinto and Lourenço-de-Oliveira ${ }^{(5)}$ evaluated An. bellator isoenzymes in populations from three locations in Brazil [Santa Catarina (SC), São Paulo (SP) and Bahia (BA)], and one from Trinidad Island (Trinidad and Tobago). They concluded that the Trinidad Island population was genetically distant from the Brazilian populations, mostly from BA. Also, regarding the three Brazilian samples, SC and SP were genetically close to each other, and quite distant from BA. Recently, a handful of studies have tried to understand the evolutionary relationship among different species from Kerteszia subgenus (including An. bellator, Anopheles cruzii and Anopheles homunculus), $)^{(6,7)}$ rather than investigating the genetic diversity between An. bellator populations. Therefore, to date, there have

\section{doi: 10.1590/0074-02760180598}

Financial support: FIOCRUZ (ANP), CNPq (grant no. 442300/2014-0) and Newton International Fellowship / Royal Society (grant no. NF161472) (LDPR).

+ Corresponding author: luisa.rona@imperial.ac.uk; luisa.rona@ufsc.br

(1) https://orcid.org/0000-0002-3400-3950

Received 18 December 2018

Accepted 3 April 2019 been no further investigations regarding the relationship between An. bellator populations from Brazil.

From the south of Brazil to the north-east, An. bellator, An. homunculus and An. cruzii are sympatric in the Atlantic Forest. ${ }^{(1,8)}$ However, while An. homunculus populations from these regions showed no clear genetic separation - they were in fact considered a single species - An. cruzii was shown to be a complex of at least two siblings: one major group with broad range dispersion from south and south-east Brazil and a distinct sibling species in BA. ${ }^{(8,9,10,11)}$ This knowledge raises an interesting question: are An. bellator populations highly structured like those from An. cruzii; or, like An homunculus, is it a species without significant genetic differentiation in its wide range distribution? To answer this question and to better understand the genetic variability and divergence among An. bellator populations, partial fragments of timeless and Clock genes were evaluated in five Brazilian locations: Ilha do Mel (PR), Cananéia (SP), Abraão and Sítio Forte (RJ) and Camacan (BA). Clock and timeless loci are involved in the circadian rhythms of insects, ${ }^{(12)}$ and have previously been shown to be good molecular markers to define different species in the An. cruzii complex..$^{(10,11,13,14)}$

\section{MATERIALS AND METHODS}

Sampling sites - The mosquitoes used in this study were captured along the Brazilian Atlantic Forest at the following localities: Ilha do Mel (PR), Cananéia (SP), Abraão and Sítio Forte (RJ), and Camacan (BA) (Fig. 1). The maps found in Fig. 1 were made using the packages maptools, maps and GISTools ${ }^{(15,16,17)}$ from R Software version 3.3.2. ${ }^{(18)}$ 
An. bellator adults and immatures were obtained, treated and preserved as described in de Rezende Dias et al. ${ }^{(14)}$ The immatures were collected in bromeliads located on rocks, in Restinga areas. Species identification was carried out according to Consoli and Lourençode-Oliveira. ${ }^{(2)}$ The detailed field collection information, such as sex and the life stage in which the samples were collected (adult or larvae), of each specimen is shown in Supplementary data (Table I).

Molecular data - The timeless and Clock partial gene sequences from the five An. bellator populations were obtained by polymerase chain reaction (PCR), cloning and sequencing as described in de Rezende Dias et al. ${ }^{(14)}$ The primers used in the PCR reactions were those described in Rona et al. ${ }^{(10,11)}$ The sequencing was carried out in an ABI3130 DNA sequencer at Myleus Biotechnology. For each mosquito, at least eight clones were sequenced to mitigate PCR errors, and allow the identification of the two alleles. Consensus sequences representing the two alleles were generated. The individuals were classified as homozygotes when only one haplotype was observed among the eight sequences. Sequences were submitted to GenBank under the Accession Numbers: MG755641 - MG755734. An alignment of all An. bellator sequences for each gene is presented in Supplementary data (Figs. 1,2).

Sequences from the timeless gene fragment from Anopheles (Kerteszia) laneanus and Anopheles (Kerteszia) cruzii s.s. were used in order to verify the relationship of An. bellator samples analysed in this study with other closely related Kerteszia species. The sequences from An. laneanus were obtained by PCR, cloning and sequencing as described above and submitted to GenBank under the Accession Numbers: KT803975 - KT803978. The sequences from An. cruzii s.s. were those previously published by Rona et al. (10) (GenBank accession numbers FJ408732, FJ408735, FJ408746, FJ408786, FJ408788, FJ408813, FJ408816, FJ408832, FJ408736, FJ408738 for SC population and FJ408838, FJ408840, FJ408848, FJ408854, FJ408855, FJ408856, FJ408862, FJ408864 for BA population).

DNA sequence analysis - Anopheles bellator DNA sequences were aligned with $\mathrm{MAFFT}^{(19)}$ and phylogenetic trees were constructed for each gene under the Maximum Likelihood method using the ape and phangorn packages ${ }^{(20,21)}$ in $\mathrm{R}$ version 3.3.2. ${ }^{(18)}$ The best-fit substitution models $\mathrm{HKY}+\mathrm{G}($ Clock) and TIM3e $+\mathrm{G}+\mathrm{I}$ (timeless) were selected following the AIC criterion using the modelTest function from phangorn. ${ }^{(21)}$ An additional timeless phylogenetic tree using the coding regions from An. bellator, An. laneanus and An. cruzii s.s. were also constructed (K80+G model) as described above.

DnaSP v5 software ${ }^{(22)}$ was used to calculate intrapopulation statistics, including nucleotide diversity and number of segregating sites, and also to ensure that all loci met the neutrality model by calculating the Tajima's $D{ }^{(23)}$ The $\mathrm{P}_{\mathrm{RO}} \mathrm{S}_{\mathrm{EQ}} \vee 2.91^{(24)}$ software was used to perform inter-population analyses, including the identification of fixed and shared polymorphisms and the generation of pair-wise estimates of population differentiation (e.g. $F_{S T}, D x y$ and $\left.D a\right)$.
A

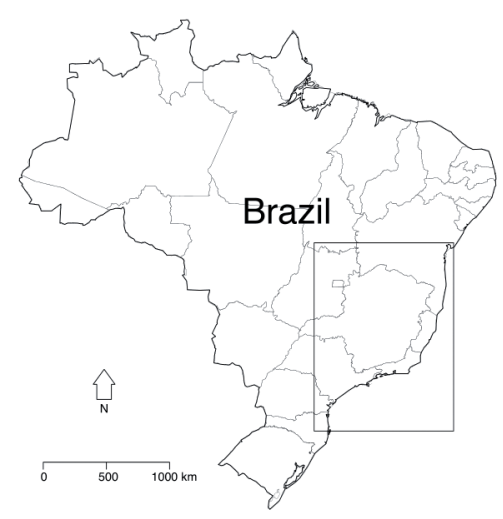

\begin{tabular}{l|c|c}
\hline Population & Latitude & Longitude \\
\hline Ilha do Mel (PR) & -25.516167 & -48.333972 \\
Cananéia (SP) & -25.016667 & -47.916667 \\
Sítio Forte (RJ) & -23.117986 & -44.274947 \\
Abraão (RJ) & -23.134761 & -44.170019 \\
Camacan (BA) & -15.391917 & -39.565167 \\
\hline
\end{tabular}

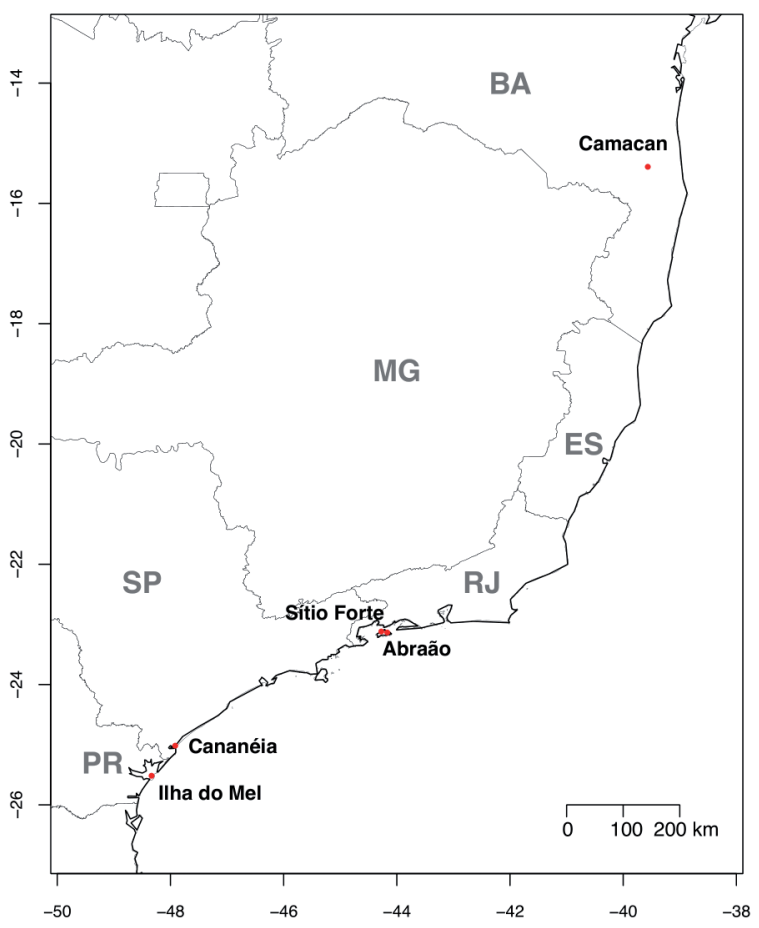

Fig. 1: Anopheles bellator collection sites in the Brazilian Atlantic Rainforest. (A): map of Brazil. (B): magnification of the black box from (A) displaying the sites where An. bellator samples were collected. The $\mathrm{x}$ and $\mathrm{y}$ axes drawn in the map (B) display the longitude and latitude, respectively. 


\section{RESULTS AND DISCUSSION}

Population structure analysis - A total of 94 sequences (two alleles from each An. bellator individual) were analysed for the two loci [Supplementary data (Tabela II)]. Clock and timeless fragment sequences were $164 \mathrm{bp}$ and $368 \mathrm{bp}$ long, respectively [Supplementary data (Figs 1,2)]. Polymorphism data from a variety of measures - e.g. nucleotide diversity ( $\theta$ and $\pi$ values) - showed that in general the timeless gene has lower genetic diversity compared with Clock [Supplementary data (Tabela II)], although the first has a bigger fragment size. None of the Tajima's D results were significant, which means that there is no evidence of natural selection acting in any of the populations [Supplementary data (Tabela II)].

In all pairwise comparisons with the BA population, very high and significant $F_{S T}$ values (ranging from 0.62 to 0.67 ) were found, there were few shared polymorphisms and a large number of fixed differences (Table), some of which caused amino acid changes [Supplementary data (Figs 1,2)]. The average number of nucleotide substitutions per site $(D x y)$ and the number of net nucleotide substitutions per site between populations $(D a)$ was also higher in the BA pairwise comparisons, confirming the $F_{S T}$ measurements. The southern and southeastern populations exhibited limited differentiation, characterized by shared polymorphisms and no fixed differences.

Haplotype genealogies analysis - The genealogical relationship of haplotypes was inferred by Maximum Likelihood for the two genes (Fig. 2). The resulting trees showed no clear separation between the sequences from PR, SP and RJ (henceforth called An. bellator A), however they clearly grouped the sequences from BA (henceforth called Anopheles bellator B) in a different cluster with high bootstrap values.

In order to verify the genealogical relationship between An. bellator A and B with other closely related Kerteszia species (An. cruzii s.s. and An. laneanus), a Maximum Likelihood tree was constructed using the coding regions of the timeless gene fragment, since the introns showed a substantial number of indels in the alignment [Supplementary data (Fig. 3)]. The resulting phylogenetic tree showed that An. bellator A and $\mathrm{B}$ are a monophyletic group, clustering them in a separated clade with high bootstrap value. The final topology was very similar to those from Foster et al. ${ }^{(25)}$ and Lorenz et al. ${ }^{(6)}$ using mitochondrial and genomic sequences: An. cruzii s.s. and An. laneanus are genetically closer when compared with An. bellator. An attempt was also made to compare An. bellator Clock sequences with those from different Kerteszia species available in the GenBank. Although, the same problem found in the timeless noncoding regions (indels) was also disclosed in the Clock alignment, but in a more challenging way, since more than $70 \%$ of the fragment is composed by an intron.

Anopheles bellator A and B - Two highly An. bellator structured groups (An. bellator A and B) were disclosed in this study. The population divergence between them is well supported by (i) fixed differences (Table), (ii) high $F_{S T}$ values (average of 0.65 ) (Table), and (iii) it is the earliest divergent node in both phylogenetic trees with strong
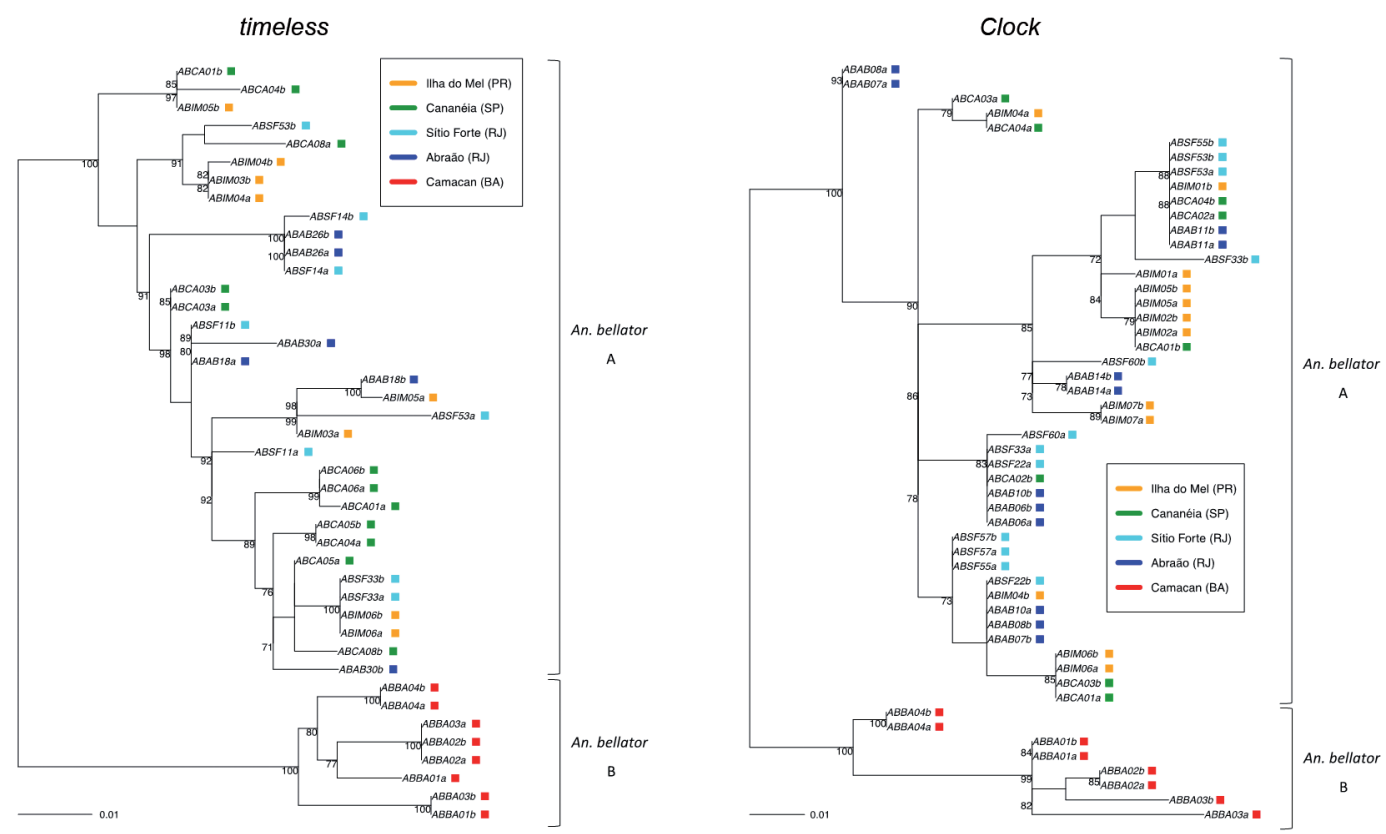

Fig. 2: maximum likelihood trees of Anopheles bellator timeless and Clock sequences (TIM3e+G+I and HKY $+\mathrm{G}$ models, respectively). These phylogenies show that two structured groups were identified in the An. bellator populations from Brazil: An. bellator B occurs in the NorthEast Atlantic Rainforest (Camacan) and An. bellator A is found in the other more Southern Brazilian regions. Numbers on the nodes represent the percentage bootstrap values based on 1000 replications. Lowercase letters in the haplotype names specify the two alleles (A or B) obtained from each individual. 
TABLE

Genetic differentiation between all Anopheles bellator populations

\begin{tabular}{|c|c|c|c|c|c|c|c|}
\hline Populations & Gene & $\mathrm{Km}$ & $F_{S T}$ & Dxy & $D a$ & Ss & $S f$ \\
\hline \multirow{2}{*}{ Abraão (RJ) x Sítio Forte (RJ) } & timeless & 10.89 & -0.1039 & 0.0177 & -0.0018 & 11 & 00 \\
\hline & Clock & & -0.0157 & 0.0322 & -0.0005 & 08 & 00 \\
\hline \multirow{2}{*}{ Ilha do Mel (PR) x Abraão (RJ) } & timeless & 496.5 & 0.0411 & 0.0215 & 0.0009 & 09 & 00 \\
\hline & Clock & & 0.1134 & 0.0404 & 0.0046 & 07 & 00 \\
\hline \multirow{2}{*}{ Ilha do Mel (PR) $x$ Sítio Forte (RJ) } & timeless & 488.6 & 0.0695 & 0.0224 & 0.0016 & 11 & 00 \\
\hline & Clock & & 0.0904 & 0.0377 & 0.0034 & 08 & 00 \\
\hline \multirow{2}{*}{ Ilha do Mel (PR) x Cananéia (SP) } & timeless & 70.1 & 0.1147 & 0.0233 & 0.0027 & 10 & 00 \\
\hline & Clock & & -0.0508 & 0.0376 & -0.0019 & 10 & 00 \\
\hline \multirow{2}{*}{ Cananéia (SP) $x$ Sítio Forte $(\mathrm{RJ})$} & timeless & 425.7 & $0.1158^{*}$ & 0.0221 & 0.0026 & 08 & 00 \\
\hline & Clock & & 0.0001 & 0.0364 & 0.0000 & 09 & 00 \\
\hline \multirow{2}{*}{ Cananéia (SP) $x$ Abraão (RJ) } & timeless & 434.1 & $0.1311^{*}$ & 0.0222 & 0.0029 & 06 & 00 \\
\hline & Clock & & 0.0086 & 0.0383 & 0.0003 & 08 & 00 \\
\hline \multirow{2}{*}{ Camacan (BA) $x$ Ilha do Mel (PR) } & timeless & 1449 & $0.6471^{*}$ & 0.0621 & 0.0402 & 00 & 07 \\
\hline & Clock & & $0.6482^{*}$ & 0.1168 & 0.0757 & 02 & 05 \\
\hline \multirow{2}{*}{ Camacan (BA) x Cananéia (SP) } & timeless & 1379 & $0.6694^{*}$ & 0.0622 & 0.0417 & 00 & 09 \\
\hline & Clock & & $0.6287^{*}$ & 0.1163 & 0.0731 & 02 & 05 \\
\hline \multirow{2}{*}{ Camacan (BA) x Abraão (RJ) } & timeless & 987.1 & $0.6722^{*}$ & 0.0628 & 0.0422 & 01 & 10 \\
\hline & Clock & & $0.6236^{*}$ & 0.1050 & 0.0655 & 02 & 03 \\
\hline \multirow{2}{*}{ Camacan (BA) $x$ Sítio Forte $(\mathrm{RJ})$} & timeless & 990.1 & $0.6758^{*}$ & 0.0641 & 0.0434 & 00 & 09 \\
\hline & Clock & & $0.6562^{*}$ & 0.1105 & 0.0725 & 02 & 05 \\
\hline
\end{tabular}

$F_{S T}$ : pair-wise estimates of population differentiation. The significance of $F_{S T}$ values was evaluated by 1,000 random permutations $(* \mathrm{p}<0.05)$. Dxy: average number of nucleotide substitutions per site between populations; $D a$ : number of net nucleotide substitutions per site between populations. ${ }^{(29)} S s$ : number of shared polymorphisms between the two populations. Sf: number of fixed differences between the two populations. Km: the approximated geographic distances between localities in $\mathrm{km}$.

statistical support (Fig. 2). The results shown here are in agreement with a former study that used allozymes as genetic markers and showed that among all pairwise comparisons between Brazilian An. bellator samples, the highest genetic distances were found between Itaparica Island (BA) and the other more southern populations from Florianópolis (SC) and Cananéia (SP). ${ }^{(5)}$ However, the BA population (Camacan) used in this study is 300 $\mathrm{Km}$ away (to the south direction) from Itaparica Island (BA), which raises new questions like: Does An. bellator from Camacan belong to the same group as An. bellator from Itaparica Island? Also, are there additional structured An. bellator groups in the Atlantic Forest? A more extensive sampling across the geographic distribution of An. bellator, mainly focusing in Espírito Santo (ES) and $\mathrm{BA}$, and the use of a larger number of molecular markers are desirable in order to better understand the evolutionary forces that establish and maintain the population structure of An. bellator. Accordingly, a number of other studies have shown the same separation pattern between northern and southern Atlantic Forest segments in many fauna (including An. cruzii s.s.) and flora groups, which converge around northern ES and southern BA, suggesting a common vicariant event. ${ }^{(10,11,26)}$
The high genetic differentiation observed between $\mathrm{BA}$ and the more southern populations might also be explained by the geographic distance between them (at least $980 \mathrm{Km}$ away) (Table). However, we would expect higher $F_{S T}$ values in the pairwise comparisons between PR and SP, with the two populations from RJ (average $F_{S T}$ of only 0.07 ), which do not have a negligible distance apart $(\sim 500 \mathrm{~km})$. Even so, among the southern and southeastern populations, we found a widespread lack of significant differentiation.

This study uncovers two structured An. bellator groups in the Brazilian Atlantic Forest: An. bellator A is widespread in Southern and Southeastern regions, and An. bellator B is found in the north-east Brazil. Interestingly, in spite of the fact that malaria cases are accounted for yearly in BA, An. bellator has never been viewed as a malaria vector in this state ${ }^{(27,28)}$ as it is for the Southern Brazilian regions. ${ }^{(2)}$ So, future studies would be valuable in order to differentiate the two An. bellator groups regarding malaria susceptibility.

\section{ACKNOWLEDGEMENTS}

To Dr Edmundo Carlos Grisard and Dr Patricia Hermes Stoco for their logistic support during this study; to Bibiana Sgorla, Clisten Fátima Staffen and Mari Dalva Staffen for 
their technical assistance; to David A Ellis for kindly reviewing the English and to Dr Rosely dos Santos Malafronte and Dr Maria Anice M Sallum for providing Anopheles bellator from Cananéia and Anopheles laneanus, respectively.

\section{AUTHORS' CONTRIBUTION}

LDPR, KV, MPCR and CLMP participated in data generation and analysis; LDPR, BWF, MANS, CJCP and ANP carried mosquito collections and morphological identification; LDPR drafted the manuscript; ANP and CJCP helped in the paper drafting by critically reading the original manuscript; LDPR was the principal investigator, participated in its design and coordination. All authors read and approved the final manuscript.

\section{REFERENCES}

1. Aragão MB. Geographic distribution and abundance of Anopheles species (Kerteszia) (Diptera, Culicidae). Rev Bras Malariol Doenças Trop. 1964; 16: 73-109.

2. Consoli RAGB, Lourenço-de-Oliveira R. Principais mosquitos de importância sanitária no Brasil. Rio de Janeiro: Fiocruz; 1994. $225 \mathrm{pp}$.

3. Smith LB. Bromeliad malaria. Annual Rep Smithson Inst. 1952; 385-98.

4. Rachou RG, Ferreira MO, Lobo AG, Pires WM. Epidemiology of malaria in Southern Brazil. Rev Bras Malariol Doenças Trop. 1955; 6: 177-88.

5. de Carvalho-Pinto CJ, Lourenço-de-Oliveira R. Isoenzymatic analysis of four Anopheles (Kerteszia) bellator Dyar \& Knab (Diptera: Culicidae) populations. Mem Inst Oswaldo Cruz. 2003; 98(8): $1045-8$.

6. Lorenz C, Patané JS, Suesdek L. Morphogenetic characterisation, date of divergence, and evolutionary relationships of malaria vectors Anopheles cruzii and Anopheles homunculus. Infect Genet Evol. 2015; 35: 144-52.

7. Oliveira TM, Foster PG, Bergo ES, Nagaki SS, Sanabani SS, Marinotti O, et al. Mitochondrial genomes of Anopheles (Kerteszia) (Diptera: Culicidae) from the Atlantic Forest, Brazil. J Med Entomol. 2016; 53(4): 790-7.

8. Cardoso JC, Bergo ES, Oliveira TMP, Sant'ana DC, Motoki MT, Sallum MAM. New records of Anopheles homunculus in Central and Serra do Mar biodiversity corridors of the Atlantic Forest, Brazil. J Am Mosq Control Assoc. 2012; 28(1): 1-5.

9. de Carvalho-Pinto CJ, Lourenço-de-Oliveira R. Isoenzymatic analysis of four Anopheles (Kerteszia) cruzii (Diptera: Culicidae) populations of Brazil. Mem Inst Oswaldo Cruz. 2004; 99(5):471-5.

10. Rona LD, Carvalho-Pinto CJ, Gentile C, Grisard EC, Peixoto AA. Assessing the molecular divergence between Anopheles (Kerteszia) cruzii populations from Brazil using the timeless gene: further evidence of a species complex. Malar J. 2009; 8: 60.

11. Rona LD, Carvalho-Pinto CJ, Mazzoni CJ, Peixoto AA. Estimation of divergence time between two sibling species of the Anopheles (Kerteszia) cruzii complex using a multilocus approach. BMC Evol Biol. 2010; 10: 91.
12. Sakai T, Ishida N. Circadian rhythms of female mating activity governed by clock genes in Drosophila. Proc Natl Acad Sci. 2001; 98(16): 9221-5.

13. Rona LDP, Carvalho-Pinto CJ, Peixoto AA. Evidence for the occurrence of two sympatric sibling species within the Anopheles (Kerteszia) cruzii complex in southeast Brazil and the detection of asymmetric introgression between them using a multilocus analysis. BMC Evol Biol. 2013; 13: 207.

14. de Rezende Dias G, Fujii TTS, Fogel BF, Lourenço-de-Oliveira R, Silva-do-Nascimento TF, Pitaluga AN, et al. Cryptic diversity in an Atlantic Forest malaria vector from the mountains of SouthEast Brazil. Parasit Vectors. 2018; 11(1): 36.

15. Brunsdon C, Chen H. GISTools: some further GIS capabilities for R. R package version 0.7-4. 2014. Available from: https:// CRAN.R-project.org/package $=$ GISTools.

16. Bivand R, Lewin-Koh N. maptools: tools for reading and handling spatial objects. R package version 0.9-2. 2017. Available from: https://CRAN.R-project.org/package=maptools.

17. Becker RA, Wilks AR, Brownrigg R, Minka TP, Deckmyn A. maps: Draw Geographical Maps. R package version 3.2.0. 2017. Available from: https://CRAN.R-project.org/package=maps.

18. R Core Team. R: a language and environment for statistical computing. R Foudation for Statistical Computing. Vienna: R Foudation for Statistical Computing; 2014.

19. Katoh K, Standley DM. MAFFT multiple sequence alignment software version 7: improvements in performance and usability. Mol Biol Evol. 2013; 30(4): 772-80.

20. Paradis E, Claude J, Strimmer K. APE: analyses of phylogenetics and evolution in R language. Bioinformatics. 2004; 20(2): 289-90.

21. Schliep KP. phangorn: phylogenetic analysis in R. Bioinformatics. 2011; 27(4): 592-3.

22. Rozas J, Sánchez-DelBarrio JC, Messeguer X, Rozas R. DnaSP, DNA polymorphism analyses by the coalescent and other methods. Bioinformatics. 2003; 19(18): 2496-7.

23. Tajima F. Statistical method for testing the neutral mutation hypothesis by DNA polymorphism. Genetics. 1989; 123(3): 585-95.

24. Filatov D. ProSeq: a software for preparation and evolutionary analysis of DNA sequence data sets. Mol Ecol Notes. 2002; 2: 621-4.

25. Foster PG, de Oliveira TMP, Bergo ES, Conn JE, Sant'Ana DC, Nagaki SS, et al. Phylogeny of Anophelinae using mitochondrial protein coding genes. R Soc Open Sci. 2017; 4(11): 170758.

26. Patterson BD, Costa LP. Bones, clones, and biomes: the history and geography of recent neotropical mammals. Chicago: University of Chicago Press; 2012. 432 pp.

27. Rachou RG. Anofelinos do Brasil: Comportamento das especies vetoras de malaria. Rev Bras Malar Doencas Trop. 1958; 10: 145-81.

28. Deane LM. Malaria vectors in Brazil. Mem Inst Oswaldo Cruz. 1986; 81(Suppl. II): 5-14.

29. Nei M, Kumar S. Molecular evolution and phylogenetics. New York: Oxford University Press; 2000. 352 pp. 